\title{
On Characteristic Poset and Stanley Decomposition
}

\author{
Sarfraz Ahmad, Imran Anwar, Ayesha Asloob Qureshi
}

\begin{abstract}
Let $J \subset I$ be two monomial ideals such that $I / J$ is Cohen Macaulay. By associating a finite posets $P_{I / J}^{g}$ to $I / J$, we show that if $I / J$ is a Stanley ideal then $\widetilde{I / J}$ is also a Stanley ideal, where $\widetilde{I / J}$ is the polarization of $I / J$. We also give relations between sdepth and fdepth of $I / J$ and $\widetilde{I / J}$.
\end{abstract}

\section{Introduction}

Let $K$ be a field and $S=K\left[x_{1}, \ldots, x_{n}\right]$ be the polynomial ring in $n$ variables. Let $J \subset I \subset S$ be two monomial ideals such that $I / J$ is a $\mathbb{Z}^{n}$-graded $S$ module. Let $u \in I / J$ be a homogeneous monomial and $Z \subseteq\left\{x_{1}, \ldots, x_{n}\right\}$. We denote by $u K[Z]$ the $K$-subspace of $I / J$ generated by all elements $u v$ where $v$ is a monomial in $K[Z]$. If $u K[Z]$ is a free $K[Z]$-module then the $\mathbb{Z}^{n}$-graded $K$-subspace $u K[Z] \subset I / J$ is called a Stanley space of dimension $|Z|$.

A Stanley decomposition of $I / J$ is a presentation of the $\mathbb{Z}^{n}$-graded $K$-vector space $I / J$ as a finite direct sum of Stanley spaces $\mathcal{D}: I / J=\bigoplus_{i=1}^{m} u_{i} K\left[Z_{i}\right]$. The Stanley depth of $I / J$ is defined to be

$$
\operatorname{sdepth}(I / J)=\max \{\operatorname{sdepth} \mathcal{D}: \mathcal{D} \text { is a Stanley decomposition of } I / J\} .
$$

Stanley [10] conjectured that there always exists a Stanley decomposition such that $\operatorname{sdepth}(I / J) \geq \operatorname{depth}(I / J)$. If the conjecture holds for some ideal $I$, then

Key Words: monomial ideals, posets, Stanley's conjecture, Stanley ideals, polarization 2010 Mathematics Subject Classification: Primary 13H10, Secondary 13C14, 13F20, $13 \mathrm{~F} 55$.

Received: December, 2012.

Accepted: April, 2013. 
we call $I$ a Stanley ideal. The conjecture is widely discussed in recent years for example [2], [4], [6], [8], [9].

Let $u$ be a monomial in $S$. Then

$$
\tilde{u}=\prod_{i=1}^{n} \prod_{j=1}^{a_{i}} x_{i j} \in T
$$

is called the polarization of $u$, where $T=K\left[x_{11}, \ldots, x_{1 a_{1}}, \ldots, x_{n 1}, \ldots, x_{n a_{n}}\right]$. Let $I$ be a monomial ideal in $S$ with monomial generators $\left(u_{1}, \ldots, u_{r}\right)$. Then the ideal generated by $\left(\tilde{u}_{1}, \ldots, \tilde{u}_{r}\right)$ is called the polarization of $I$ and is denoted by $\tilde{I}$. For more details, see [3]. As a main result of this paper we show that if $I / J$ is a CM Stanley ideal, then $\widetilde{I / J}$ is also a CM Stanley ideal by using a more appropriate approach than [1]. We use the idea of characteristic poset from [6]. A partial order on $\mathbb{N}^{n}$ is given by $(a(1), \ldots, a(n)) \leq(b(1), \ldots, b(n))$ if $a(i) \leq b(i)$ for all $i$. Let $g \in \mathbb{N}^{n}$ be an integer vector with the property that $a \leq g$ for all $a \in \mathbb{Z}^{n}$ with $x^{a} \in I / J$. Here $x^{a}$ denote the monomial $x_{1}^{a(1)} \ldots x_{n}^{a(n)}$ where $a=(a(1), \ldots, a(n)) \in \mathbb{N}^{n}$. The characteristic poset (see [6]) $P_{I / J}^{g}$ of $I / J$ with respect to $g$ is the subposet of $\mathbb{N}^{n}$ given by

$$
P_{I / J}^{g}=\left\{a \in \mathbb{Z}^{n}: x^{a} \in I \backslash J, \quad a \leq g\right\}
$$

Each Stanley decomposition of $I / J$ gives a partition of $P_{I / J}^{g}$ and vice versa. We call a partition of $P_{I / J}^{g}$, a nice partition if its corresponding Stanley decomposition satisfies Stanley's conjecture. In Proposition 1.2, we give a necessary and sufficient condition for a partition to be nice. In Theorem 1.5, we show that if $P_{I / J}$ has a nice partition, then $P_{\widetilde{I / J}}$ also has a nice partition.

In [6], the concept of fdepth is introduced which is a natural lower bound for sdepth and depth. It is defined as

$$
\text { fdepth } M=\max \{\text { fdepth } \mathcal{F}: \mathcal{F} \text { is a prime filtration of } M\} \text {. }
$$

In Corollary 1.6, we show that sdepth (fdepth) of $\widetilde{I / J}$ can be computed by computing sdepth (fdepth) of $I / J$.

\section{Posets and their Partitions}

The natural partial order $\leq$ on $\mathbb{N}^{n}$ is defined as follows: $a \leq b$, with $a=$ $(a(1), \ldots, a(n))$ and $b=(b(1), \ldots, b(n))$ if and only if $a(i) \leq b(i)$ all for $i=$ $1, \ldots, n$. The meet $a \wedge b$ and join $a \vee b$ with respect to $\leq$ are $(\min \{a(1), b(1)\}, \ldots, \min \{a(n), b(n)\})$ and $(\max \{a(1), b(1)\}, \ldots, \max \{a(n), b(n)\})$, 
respectively.

With this natural partial order $\mathbb{N}^{n}$ is a distributive lattice.

Let $K$ be a field and $S=K\left[x_{1}, \ldots, x_{n}\right]$ be the polynomial ring in $n$ variables. For any $c=(c(1), \ldots, c(n)) \in \mathbb{N}^{n}$ we denote by $x^{c}$ the monomial $x_{1}^{c(1)} \cdots x_{n}^{c(n)}$. Let $I$ and $J$ be two monomial ideals in $S$ such that $J \subset I$. Let $I=\left(x^{a_{1}}, \ldots, x^{a_{r}}\right)$ and $J=\left(x^{b_{1}}, \ldots, x^{b_{s}}\right)$ for some $a_{i}, b_{i} \in \mathbb{N}^{n}$ for all $i$. We associate a poset to $I / J$ in the following way: we choose $g \in \mathbb{N}^{n}$ such that $a_{i} \leq g$ and $b_{j} \leq g$ for all $i$ and $j$. Let $P_{I / J}^{g}$ be the set of all $c \in \mathbb{N}^{n}$ with $c \leq g$ and such that $a_{i} \leq c$ for some $i$ and $c \nsupseteq b_{j}$ for all $j$. The set $P_{I / J}^{g}$ is a finite subposet in $\mathbb{N}^{n}$ and called (see [6]) the characteristic poset of $I / J$ with respect to $g$. A natural choice for $g$ is the join of all the $a_{i}$ and $b_{j}$. For this choice of $g$, the poset $P_{I / J}^{g}$ has the least number of elements.

Given any poset $P$ and $a, b \in P$, an interval $[a, b]$ is defined as $[a, b]=\{c \in$ $P: a \leq c \leq b\}$. Suppose $P$ is a finite poset. A partition of $P$ is a disjoint union

$$
\mathcal{P}: \quad P=\bigcup_{i=1}^{r}\left[a_{i}, b_{i}\right]
$$

of intervals of $P$.

In order to describe the Stanley decomposition of $I / J$ coming from a partition of $P_{I / J}^{g}$ we adopt the following notation from [6]: for each $b \in P_{I / J}^{g}, Z_{b}$ is the set $\left\{x_{j}: b(j)=g(j)\right\}$. The function $\rho$ is introduced as

$$
\rho: P_{I / J}^{g} \rightarrow \mathbb{Z}_{\geq 0}, \quad c \mapsto \rho(c),
$$

where $\rho(c)=|\{j: c(j)=g(j)\}|\left(=\left|Z_{c}\right|\right)$. Now we quote the following theorem from $[6]$.

Theorem 1.1. Let $\mathcal{P}: P_{I / J}^{g}=\bigcup_{i=1}^{r}\left[c_{i}, d_{i}\right]$ be a partition of $P_{I / J}^{g}$. Then

$$
\mathcal{D}(\mathcal{P}): I / J=\bigoplus_{i=1}^{r}\left(\bigoplus_{c} x^{c} K\left[Z_{d_{i}}\right]\right)
$$

is a Stanley decomposition of $I / J$, where the inner direct sum is taken over all $c \in\left[c_{i}, d_{i}\right]$ for which $c(j)=c_{i}(j)$ for all $j$ with $x_{j} \in Z_{d_{i}}$. Moreover, $\operatorname{sdepth} \mathcal{D}(\mathcal{P})=\min \left\{\rho\left(d_{i}\right): i=1, \ldots, r\right\}$.

It is also shown in $[6$, Theorem 2.4] that sdepth $I / J$ can be computed as the maximum of the numbers sdepth $\mathcal{D}(\mathcal{P})$, where $\mathcal{P}$ runs over the (finitely many) partitions of $P_{I / J}^{g}$. From these results we conclude that Stanley's conjecture holds for $I / J$ if and only if there exists a partition $\mathcal{P}: P_{I / J}^{g}=\bigcup_{i=1}^{t}\left[a_{i}, b_{i}\right]$ of the 
poset $P$ such that

$$
\left|\rho\left(b_{i}\right)\right| \geq \operatorname{depth}(I / J) \text { for all } i .
$$

Any partition of a poset satisfying condition (1.2) will be called nice.

To this end, we give some definitions associated to a poset. Let $P$ be a finite poset. An element $m \in P$ is called a maximal element if there is no $a \in P$ with $a>m$. We denote by $\mathcal{M}(P)$ the set of maximal elements of $P$. An element $a \in P$ is called a facet of $P$ if for all $m \in \mathcal{M}(P)$ with $a \leq m$ one has $\rho(a)=\rho(m)$. The set of all facets of $P$ will be denoted by $\mathcal{F}(P)$.

A chain $\mathfrak{F}: 0=M_{0} \subset M_{1} \subset \cdots \subset M_{m}=M$ of $\mathbb{Z}^{n}$-graded submodules of $M$ is called a prime filtration of $M$ if $M_{i} / M_{i-1} \cong\left(S / P_{i}\right)\left(-a_{i}\right)$ where $a_{i} \in$ $\mathbb{Z}^{n}$ and where each $P_{i}$ is a monomial prime ideal. The set of prime ideals $\left\{P_{1}, \ldots, P_{m}\right\}$ is called the support of $\mathfrak{F}$ and denoted by supp $\mathfrak{F}$.

The next proposition gives a necessary and sufficient condition for a partition $\mathcal{P}$ of $P_{I / J}^{g}$ to be nice.

Proposition 1.2. Let $J \subset I$ be two monomial ideals of $S$ such that $I / J$ is Cohen-Macaulay. Let $P_{I / J}^{g}$ be the poset associated to $I / J$ and $\mathcal{P}: P_{I / J}^{g}=$ $\bigcup_{i=1}^{t}\left[a_{i}, b_{i}\right]$ be a partition of $P_{I / J}^{g}$. Then the following conditions are equivalent.

(a) $\mathcal{P}$ is nice.

(b) $\left\{b_{1}, \ldots, b_{t}\right\} \subseteq \mathcal{F}\left(P_{I / J}^{g}\right)$

(c) $\mathcal{M}\left(P_{I / J}^{g}\right) \subseteq\left\{b_{1}, \ldots, b_{t}\right\} \subseteq \mathcal{F}\left(P_{I / J}^{g}\right)$

Proof. (a) $\Rightarrow$ (b): Since $I / J$ is Cohen-Macaulay therefore $|\rho(b)| \leq \operatorname{depth}(I / J)$ for all faces of $P_{I / J}^{g}$, and $|\rho(b)|=\operatorname{depth}(I / J)$ if and only if $b$ is a facet. Thus $\mathcal{P}$ is nice only if $\left\{b_{1}, \ldots, b_{t}\right\} \subseteq \mathcal{F}\left(P_{I / J}^{g}\right)$.

(b) $\Rightarrow$ (c): Let $m \in \mathcal{M}\left(P_{I / J}^{g}\right)$ and since $\mathcal{P}$ is partition of $P_{I / J}^{g}$, so $m \in\left[a_{i}, b_{i}\right]$ for some $i$. Since $m \leq b_{i}$ and $m$ is maximal, it follows that $m=b_{i}$. Thus $\mathcal{M}\left(P_{I / J}^{g}\right) \subseteq\left\{b_{1}, \ldots, b_{t}\right\}$.

$(c) \Rightarrow($ a): Let $\mathfrak{F}$ be a prime filtration of $I / J$. Then $\{\operatorname{dim} S / P: P \in$ $\operatorname{Ass}(I / J))\}=\left\{\rho\left(b_{i}\right): b_{i} \in \mathcal{F}\left(P_{I / J}^{g}\right)\right\}$. Therefore,

$$
\begin{aligned}
\min \left\{\rho\left(b_{i}\right): b_{i} \in \mathcal{F}\left(P_{I / J}^{g}\right)\right\} & =\min \left\{\rho\left(m_{j}\right): m_{j} \in \mathcal{M}\left(P_{I / J}^{g}\right)\right\} \\
& =\min \left\{\operatorname{dim}\left(S / P_{j}\right): P_{j} \in \operatorname{Ass}(I / J)\right\} \\
& \geq \operatorname{depth}(I / J) .
\end{aligned}
$$

The first equation follows from the definition of the facets, while the last inequality is a basic fact of commutative algebra. Therefore our given partition is nice. 
Remark 1.3. In the above Proposition if $\mathcal{P}$ is nice then we can refine it in such a way that for the refinement

$$
\mathcal{P}^{\prime}: P_{I / J}^{g}=\bigcup_{i=1}^{t^{\prime}}\left[a_{i}^{\prime}, b_{i}^{\prime}\right]
$$

we have $\left\{b_{1}^{\prime}, \ldots, b_{t^{\prime}}^{\prime}\right\}=\mathcal{F}\left(P_{I / J}^{g}\right)$. To prove this fact, let $\hat{b}$ be a facet such that $\hat{b} \neq b_{i}^{\prime}$, for all $i$. So there exist some interval $\left[a_{i}, b_{i}\right]$ with $\hat{b} \in\left[a_{i}, b_{i}\right]$. Since $\hat{b}$ is a facet, we obtain $\rho(\hat{b})=\rho\left(b_{i}\right)$, and there exist $j$ such that $\hat{b}(j)<b_{i}(j)$. We set $a_{i}^{\prime}(j)=\hat{b}(j)+1$ and $a_{i}^{\prime}(k)=a_{i}(k)$ for all $k$ different from $j$. The interval $\left[a_{i}, b_{i}\right]$ in $\mathcal{P}$ can be replaced by two disjoint intervals, namely $\left[a_{i}, \hat{b}\right]$ and $\left[a_{i}^{\prime}, b_{i}\right]$ and obtain the desired conclusion.

Let $u$ be a monomial in $S$. Then $\tilde{u}=\prod_{i=1}^{n} \prod_{j=1}^{a_{i}} x_{i j} \in T$ is called the polarization of $u$, where $T=K\left[x_{11}, \ldots, x_{1 a_{1}}, \ldots, x_{n 1}, \ldots, x_{n a_{n}}\right]$. Let $I \subset S$ be a monomial ideal such that $I=\left(u_{1}, \ldots, u_{r}\right)$. Then the ideal generated by $\left(\tilde{u}_{1}, \ldots, \tilde{u}_{r}\right)$ is called a polarization of $I$ and is denoted by $\tilde{I}$. We may assume that for each $i \in[n]$ there exists $j$ such that $x_{i}$ divides $u_{j}$. Let $u_{j}=x_{1}^{a_{j 1}} \cdots x_{n}^{a_{j n}}$ for $j=1, \ldots, s$ and set $r_{i}=\max a_{j i}: j=1, \ldots, s$ for $i=1, \ldots, n$. Moreover we set $r=\sum_{i=1}^{n} r_{i}$. Then $\tilde{I}$ is a squarefree monomial ideal in the polynomial $\operatorname{ring} T$ in $r$ variables. It is known that $I$ is Cohen-Macaulay if and only if $\tilde{I}$ is Cohen-Macaulay.

We denote the posets associated with $I / J$ and $\widetilde{I / J}$ by $P_{I / J}^{g}$ and $P_{\overline{I / J}}^{g^{\prime}}$, and the set of facets of $P_{I / J}^{g}$ and $P_{I / J}^{g^{\prime}}$ by $\mathcal{F}\left(P_{I / J}^{g}\right)$ and $\mathcal{F}\left(P_{\overline{I / J}}^{g^{\prime}}\right)$. Note that $\mathcal{F}\left(P_{I / J}^{g}\right)$ is a subset of the set

$$
B=\left\{b \in \mathbb{N}^{n}: b(i)<r_{i} \text { if } b(i) \neq g(i)\right\} .
$$

In order to formulate the main theorem of this paper, we introduce the following notion. We define the map $\varphi: B \rightarrow N^{r}$ as follows:

$$
b \mapsto b^{\prime}(i j)= \begin{cases}0, & \text { if } b(i)<g(i) \text { and } j=b(i)+1, \\ g^{\prime}(i j), & \text { otherwise. }\end{cases}
$$

The components of the vectors $b^{\prime}$ are indexed by pairs of numbers $i j$ and for each $i=1, \ldots, n$ the second index $j$ runs in the range $j=1, \ldots, r_{i}$.

The set of facets of a poset can be viewed as the set of facets of a multicomplex $\Gamma$ in the following way: if $a$ is a facet of $\Gamma$ with $a(i)=\infty$, then we set $a(i)=g(i)$. For details about multicomplex, see [5]. From the result of Soleyman Jahan [7, Proposition 3.8], we see that the restriction of the map $\varphi$ 
to $\mathcal{F}\left(P_{I / J}^{g}\right)$ is a bijection between the set of facets of $P_{I / J}^{g}$ and the set of facets of $P \frac{g^{\prime}}{I / J}$.

Consider the set $A=\left\{a \in \mathbb{N}^{n}: a(i) \leq r_{i}\right\}$ and the map $\psi: A \rightarrow\{0,1\}^{r}$ defined as

$$
\psi(a)(i j)= \begin{cases}0, & \text { if } j>a(i), \\ 1, & \text { otherwise. }\end{cases}
$$

The map $\psi$ is injective, because if for any $a \neq a^{\prime}$ there exists some $i$ such that $a(i) \neq a^{\prime}(i)$, say $a(i)<a^{\prime}(i)$. Then by definition of $\psi, a(i j)=0$ for $j=a(i)+1$ and $a^{\prime}(i j)=1$ for $j=a(i)+1$.

Let $I=[a, b] \subset \mathbb{N}^{n}$ be an interval such that $a=(a(1), a(2), \ldots, a(n))$ and $b=(b(1), b(2), \ldots, b(n))$. An $i$-subinterval of $I$ is defined as $\{c \in \mathbb{N}: a(i) \leq$ $c \leq b(i)\}$ and is denoted it by $I(i)=[a(i), b(i)]$. Next we quote the following Lemma from [1].

Lemma 1.4. Let $I_{1}, I_{2}$ be two intervals of a poset $P$ such that $I_{1}=[a, b]$ and $I_{2}=[c, d]$. Suppose $I_{1} \cap I_{2}=\emptyset$, then $I_{1}(i) \cap I_{2}(i)=\emptyset$, for some $i \in\{1, \ldots, n\}$

Now we state the following theorem.

Theorem 1.5. Let $I \subset J \subset S$ be two monomial ideals of $S$ such that $I / J$ is Cohen-Macaulay and $\widetilde{I / J}$ be the polarization of $I / J$. Also let $P_{I / J}^{g}$ and $P_{I / J}^{g^{\prime}}$ be the characteristic posets associated to $I / J$ and $\widetilde{I / J}$, respectively. If $P_{I / J}^{g}$ has a nice partition then $P_{\frac{g^{\prime}}{I / J}}$ has also a nice partition.

Proof. Let $P_{I / J}^{g}$ has a nice partition say $\mathcal{P}^{\prime}$. As described in Remark 1.3 we can refine the partition $\mathcal{P}^{\prime}$ to a refined partition say $\mathcal{P}:=\bigcup_{i=1}^{t}\left[c_{i}, d_{i}\right]$ such that $\left\{d_{1}, \ldots, d_{t}\right\}=\mathcal{F}(P)$. We will show that $\hat{\mathcal{P}}:=\bigcup_{i=1}^{t}\left[\hat{c}_{i}, \hat{d}_{i}\right]$ is a nice partition of $P \mathrm{~g}_{\overline{I / J}}^{g^{\prime}}$, where $\psi\left(c_{i}\right)=\hat{c}_{i}$ and $\varphi\left(d_{i}\right)=\hat{d}_{i}$ for all $i=1, \ldots, t$.

First, we show that $\left[\hat{c}_{i}, \hat{d}_{i}\right] \cap\left[\hat{c}_{j}, \hat{d}_{j}\right]=\emptyset$, for all $i \neq j$. Suppose that there exist a face $c \in\left[\hat{c}_{i}, \hat{d}_{i}\right] \cap\left[\hat{c}_{j}, \hat{d}_{j}\right] \neq \emptyset$ for some $i \neq j$. Injectivity of $\psi$ gives $\hat{c}_{i} \neq \hat{c}_{j}$. Since $\left[c_{i}, d_{i}\right] \cap\left[c_{j}, d_{j}\right]=\emptyset$, we apply Lemma 1.4 and obtain $\left[c_{i}(l), d_{i}(l)\right] \cap\left[c_{j}(l), d_{j}(l)\right]=\emptyset$ for some $l \in\{1, \ldots, n\}$. It shows that at least one of $d_{i}(l), d_{j}(l)$ is not equal to $g(l)$ say $d_{i}(l) \neq g(l)$. Then by Theorem 1.1 $b_{i}(l)=a_{i}(l)$. If $d_{j}(l)=g(l)$ and $c_{i}(l)>c_{j}(l)$ then $\left[c_{i}(l), d_{i}(l)\right] \subset\left[c_{j}(l), d_{j}(l)\right]$ which is not possible so we may assume that $c_{i}(l)<c_{j}(l)$. On the other hand if $d_{j}(l) \neq g(l)$, then we may change $i$ by $j$. Thus we can assume let $c_{i}(l)=d_{i}(l)=k-1$ and $c_{j}(l)=m>k-1$. Then by definition of $P_{I / J}^{g}$ and $\varphi$ we have $\hat{c}_{i}(l k)=0=\hat{d}_{i}(l k)$ and $\hat{c}_{j}(l l)=1$ for $l \leq m$. Thus $\hat{c}_{j}(l k)=1$. It follows 
that $c(l k)=0$. On the other hand, since $c \geq \hat{c}_{j}$, we get $c(l k) \geq \hat{c}_{j}(l k)=1$ and we obtain a contradiction to our assumption that $\left[\hat{c}_{i}, \hat{d}_{i}\right] \cap\left[\hat{c}_{j}, \hat{d}_{j}\right] \neq \emptyset$.

Now for the second part of the proof, we will use the Hilbert series. We have $H(S / I)=\sum_{i=1}^{t} s^{\left|c_{i}\right|} /(1-s)^{\rho\left(d_{i}\right)}$. The definition of $\psi$ implies that $\rho\left(c_{i}\right)=\rho\left(\hat{c}_{i}\right)$ for all $i=\{1, \ldots, t\}$. We know that the depth of $I / J$ increases by 1 for each polarization step. Also, we observe from the definition of $\varphi$ that for each polarization step, $\rho\left(d_{i}\right)$ increases by 1 . Therefore, after $p$ polarization steps $\rho\left(\hat{d}_{i}\right)=\rho\left(d_{i}\right)+p$ and

$$
H\left(\bigcup_{i=1}^{t}\left[\hat{c}_{i}, \hat{d}_{i}\right]\right)=\sum_{i=1}^{t} \frac{s^{\rho\left(c_{i}\right)}}{(1-s)^{\rho\left(d_{i}\right)+p}}=\frac{1}{(1-s)^{p}} H(S / I)
$$

is the Hilbert series of $H(\widetilde{I / J})$. Hence $\widetilde{I / J}=\bigcup_{i=1}^{t}\left[\hat{c}_{i}, \hat{d}_{i}\right]$. Note that $\bigcup_{i=1}^{t}\left[\hat{c}_{i}, \hat{d}_{i}\right]$ is a nice partition because $\rho\left(\hat{d}_{i}\right)=\rho\left(d_{i}\right)+p \geq \operatorname{depth}_{S}(S / I)+p=$ $\operatorname{depth}_{T}(\widetilde{I / J})$, for all $i$.

The converse of Theorem 1.5 is still open. We recall the definition of fdepth from [6]. Let $\mathfrak{F}$ be a prime filtration of $I / J$. Furthermore, fdepth $\mathfrak{F}=$ $\min \{\operatorname{dim} S / P: P \in \operatorname{supp} \mathfrak{F}\}$ and

fdepth $M=\max \{$ fdepth $\mathfrak{F}: \mathfrak{F}$ is a prime filtration of $M\}$.

It is not obvious how to compute the fdepth of a module, but it is very easy to see that fdepth $M \leq \operatorname{depth} M$, sdepth $M$.

Corollary 1.6. With same notation as above, we have

(a) $\operatorname{sdepth}(\widetilde{I / J})=\operatorname{sdepth}(I / J)+r-n$,

(b) $\operatorname{fdepth}(\widetilde{I / J}) \geq \operatorname{fdepth}(I / J)$.

Where $r$ and $n$ are the number of variables of $T$ and $S$, respectively.

Proof. Let $c \in \mathcal{F}\left(P_{I / J}^{g}\right)$ and $\varphi(c) \in \mathcal{F}\left(P \frac{g^{\prime}}{I / J}\right)$. Since $\operatorname{sdepth}($ fdepth) of a Stanley decomposition of $I / J$ is minimum of the numbers $\rho(c)$, the assertion follows by observing that $\rho(\varphi(c))=\rho(c)+r-n$.

\section{References}

[1] S. Ahmad, Stanley decompositions and polarization, Czechoslovak Mathematical Journal, vol. 61, no. 2 (2011), pp. 483-493. 
[2] I. Anwar and D. Popescu, Stanley conjecture in small embedding dimension, J. Alg. 318(2007), 1027-1031. Zbl 1132.13009

[3] J. Herzog, T. Hibi, Monomial Ideals, Springer, 2011.

[4] J. Herzog, A. Soleyman Jahan, S. Yassemi, Stanley decompositions and partitionable simplicial complexes, J. Algebraic Combinatorics, 27 (2008), 113-125. Zbl 1131.13020

[5] J. Herzog, D. Popescu, Finite filtrations of modules and shellable multicomplexes, Manuscripta Math. 121, (2006), 385-410. Zbl 1107.13017

[6] J. Herzog, M. Vladoiu and X. Zheng, How to compute the Stanley depth of a monomial ideal, Journal of Algebra, 322 (2009), 3151-3169. Zbl pre05658663

[7] A. Solyman Jahan, Prime filtrations of monomial ideals and polarizations, J. Algebra 312(2007), 1011-1032.

[8] S. Nasir, Stanley decomposition and localization, Bull. Math. Soc. Sc. Math. Roumanie 51(99), no.2(2008), 151-158.

[9] D. Popescu, Stanley depth of Multigraded modules, J. Algebra 321(2009), 2782-2797. Zbl 1179.13016

[10] R. P. Stanley, Linear Diophantine equations and local cohomology, Invent. Math.68, (1982), 175-193. Zbl 0516.10009

Sarfraz Ahmad,

Department of Mathematics,

COMSATS Institute of Information Technology,

M. A. Jinnah Campus, Raiwand Road, Lahore, Pakistan.

Email: sarfrazahmad@ciitlahore.edu.pk

Imran Anwar,

Department of Mathematics,

COMSATS Institute of Information Technology,

M. A. Jinnah Campus, Raiwand Road, Lahore, Pakistan.

Email: imrananwar@ciitlahore.edu.pk

Ayesha Asloob Qureshi,

Department of Mathematics,

The Abdus Salam International Center of Theocratical Physics,

Trieste, Italy.

Email: ayesqi@gmail.com 